\title{
Maximum Neutrino Mass Drops Again
}

\author{
The KATRIN experiment has delivered the best estimate to date for the \\ maximum mass of a neutrino, finding it to be over $25 \%$ lower than the \\ previous estimate.
}

By Matteo Rini

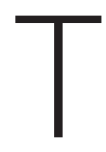
he Universe bristles with neutrinos, one of the most abundant known particles. Despite the particles' profusion, scientists still know little about neutrinos, which interact feebly with matter and are thus hard to detect and characterize. Even their mass, a most basic property, remains unknown. Now, the frontrunner experiment in the race to directly measure neutrino mass, the Karlsruhe Tritium Neutrino (KATRIN) experiment in Germany, has achieved record-breaking sensitivity to the particle's mass, finding that its value must be below $0.8 \mathrm{eV}$. The team behind the experiment says that KATRIN should soon be able to test much lower mass values, not far from those suggested by cosmological observations.

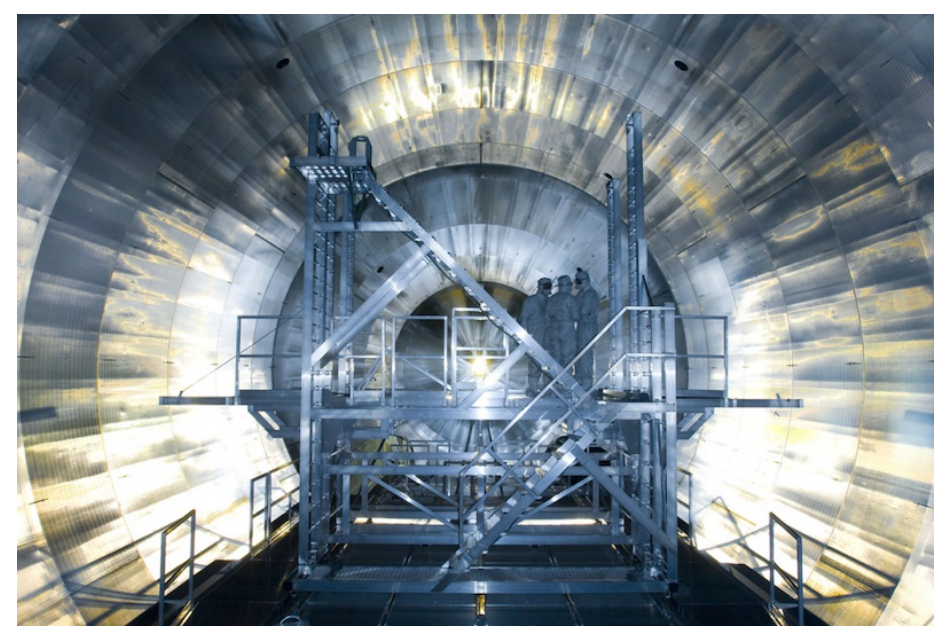

A photograph of the interior of the spectrometer used by KATRIN, an instrument designed to measure the neutrino's mass.

Credit: KATRIN
Scientists have known that neutrinos have a mass since the 1998 discovery of neutrino oscillations-a switching of their "flavor," which is only possible if their mass isn't zero. Physicists have long been trying to pin down that mass value, as well as to understand why the particle has mass. This information could expose physics beyond the standard model and is crucial for cosmology-while featherlight, these particles are so abundant that their gravitational pull could affect the formation of large cosmic structures.

Weighing the neutrino, however, is no easy feat. Today's method of choice stems from a 1934 proposal by Enrico Fermi that exploits a radioactive process called the nuclear beta decay. In this decay, a neutron transforms into a proton, releasing an electron and a type of neutrino called an antineutrino. If the neutrino has a mass, it will take on some of the energy released by the decay, limiting the maximum energy of the released electron. This effect should modify the high-energy tail of the electron spectrum, offering a way to determine the neutrino mass.

KATRIN was designed to be the most sensitive experiment of this kind, employing the world's most intense source of beta decays from tritium (a heavy isotope of hydrogen), a giant, airship-shaped spectrometer with exceptional electron-energy resolution, and tricks for reducing unwanted background signals generated by radioactive contaminants. Conceived in 2001, and fully commissioned in 2018, KATRIN ran its first measuring campaign in 2019. In only four weeks of data taking, it obtained an upper mass bound of $1.1 \mathrm{eV}$-halving the ceiling determined by previous measurements (see Viewpoint: Homing in on the Neutrino Mass). 


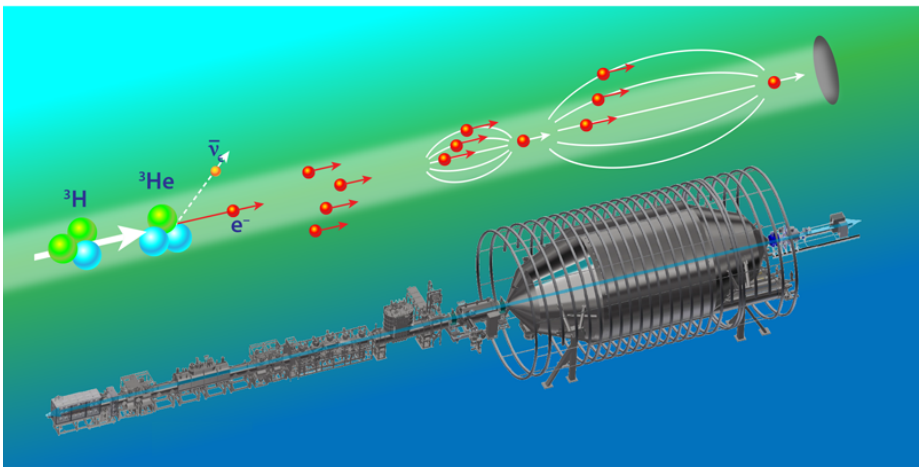

Artistic sketch of the KATRIN experiment. Tritium isotopes undergo beta decay and release electrons and neutrinos in the process. The electrons are then transported to a giant spectrometer that measures their energy spectrum. The shape of this spectrum depends on the neutrino mass.

Credit: KATRIN; adapted by APS/Alan Stonebraker

KATRIN has now reduced that value to $0.8 \mathrm{eV}$, using data from its second measurement campaign, which had "a stronger signal, lower background [levels], and ran for a longer time," says Magnus Schlösser of the Karlsruhe Institute of Technology, Germany, a co-coordinator of KATRIN's data analysis team. To boost the signal, the team increased the activity of the tritium source-the number of atoms decaying per second. (In the first run, the activity was kept low to protect the surfaces of materials exposed to tritium for the first time). To decrease the background, the team reduced radioactive contamination by improving the vacuum in the spectrometer and by stripping contaminants from surfaces through heat treatments. In addition to measures boosting the signal statistics, they also lowered systematic uncertainties associated with inaccurate modeling of experimental components, such as the source electric potential, which can distort the measured electron-energy spectrum.
Analyzing the second-run data, the team placed an upper bound of $0.9 \mathrm{eV}$ on the neutrino mass. They reduced that value by $0.1 \mathrm{eV}$ by adding into their analysis the first-run result.

The drop from $1.1 \mathrm{eV}$ to $0.8 \mathrm{eV}$ may sound small. But for Deborah Harris, an experimental physicist at York University, Canada, those two numbers alone don't paint a full picture of KATRIN's progress. She says that the impressive feat is that the KATRIN team slashed statistical uncertainties by a factor of 3-the equivalent of taking 9 times more data-and systematic uncertainties by a factor of 2 . "This [improvement] is the big news."

Particle physicist Riccardo Bugnera of the University of Padua in Italy says that the KATRIN result is "expected, but very important." The progress suggests that the collaboration can still improve this complex experiment, which bodes well for KATRIN's ultimate goal-reaching a $0.2-\mathrm{eV}$ mass sensitivity by 2024, he says.

With such a sensitivity, KATRIN would have mass-testing capabilities approaching those of indirect methods that infer the neutrino mass from cosmological observables such as the cosmic microwave background. Those methods indicate that the neutrino has a mass slightly smaller than $0.2 \mathrm{eV}$, but the value they derive depends on many cosmological assumptions. "It'd be very exciting to reach comparable sensitivity through a method that doesn't require assumptions about how the Universe works," Harris says.

Matteo Rini is the Editor of Physics. 